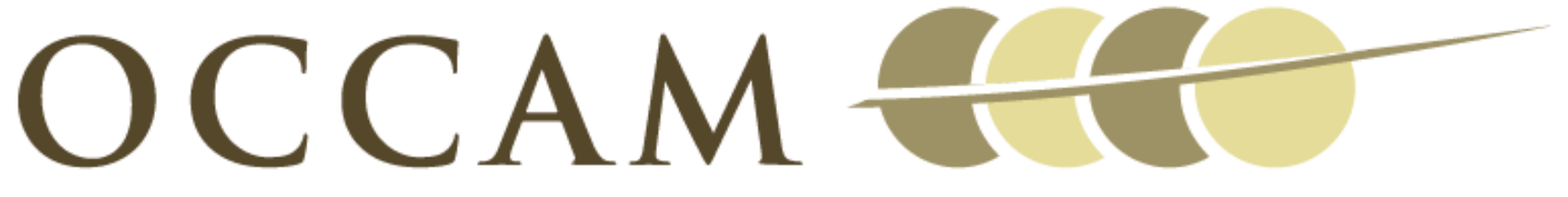

OXFORD CENTRE FOR COLLABORATIVE APPLIED MATHEMATICS

Report Number 10/09

Anticavitation and differential growth in elastic shells

$$
\text { by }
$$

D.E. Moulton , A. Goriely

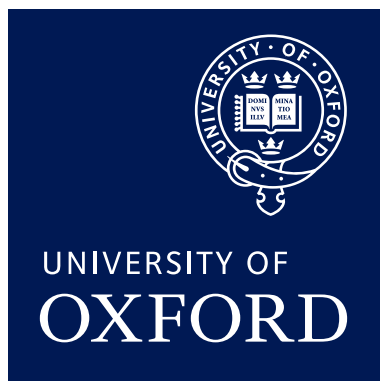

Oxford Centre for Collaborative Applied Mathematics Mathematical Institute

24 - 29 St Giles'

Oxford

OX1 3LB

England 

Journal of Elasticity manuscript No.

(will be inserted by the editor)

\title{
Anticavitation and differential growth in elastic shells
}

\author{
D.E. Moulton · A. Goriely
}

Received: date / Accepted: date

\begin{abstract}
Elastic anticavitation is the phenomenon of a void in an elastic solid collapsing on itself. Under the action of mechanical loading alone, very few materials admit anticavitation. We study the possibility of anticavitation as a consequence of an imposed differential growth. Working in the geometry of a spherical shell, we seek radial growth functions which cause the shell to deform to a solid sphere. It is shown, surprisingly, that most materials do not admit full anticavitation, even when infinite growth or resorption is imposed at the inner surface of the shell. However, void collapse can occur in a limiting sense when radial and circumferential growth are properly balanced. Growth functions which diverge or vanish at a point arise naturally in a cumulative growth process.
\end{abstract}

Keywords elasticity $\cdot$ Differential growth $\cdot$ anticavitation $\cdot$ cavitation

\section{Introduction}

Elastic cavitation is the phenomenon in which a cavity or void appears in the interior of an elastic body. This problem has received considerable interest in the literature, starting with the pioneering experimental work of Gent and Lindley [6] and the seminal analysis of Ball [3]. Ball considered solid elastic spheres under uniform hydrostatic loads and found the critical pressure at which a branch of radially symmetric configurations with an internal cavity bifurcates from the undeformed configuration. Since then, cavitation has received considerable attention and has been considered for various situations - compressibility versus incompressibility, anisotropy, and composite layers have all been analyzed in different combinations for various materials $([15,9,17,18,14,5,10]$, among others). In these studies, cavitation occurs as a consequence of external mechanical loading. More recently, several authors $[13,11]$, have shown that growth or swelling can also induce cavitation and cavitation has been suggested as a mechanism for stem hollowing in plants [8].

Derek E. Moulton

Department of Mathematics, University of Arizona, Tucson, AZ E-mail: moulton@math.arizona.edu

Alain Goriely

OCCAM, Institute of Mathematics, University of Oxford, UK;

Department of Mathematics, University of Arizona, Tucson, USA.

E-mail: goriely@math.arizona.edu 
Given far less consideration, and the subject of the present paper, is the "reverse problem" of anticavitation or void collapse. That is, under what conditions would a pre-existing cavity close on itself. The collapse of a cavity is a common occurrence in fluids [4]. In nonlinear elasticity, however, it has scarcely been studied. One analysis was conducted by Abeyaratne and Hou [1]. Following Ball's analysis of the critical pressure on a solid sphere to induce cavity formation, Abeyaratne and Hou explored the critical pressure on spherical shells needed to collapse the inner void. In doing so, they found a necessary and sufficient condition on the strain-energy function of the material such that void collapse can occur under sufficient loading. Surprisingly, most commonly used elastic materials do not satisfy the condition - for these materials, anticavitation through external pressure alone is impossible, even though cavitation may be possible. It seems to be easier to open a cavity in a sphere than close a cavity in a shell.

In this paper, we study a growing elastic body containing a cavity or void in its reference unstressed configuration and explore the possibility of collapse and disappearance of the void as a function of the material and form and rate of growth. In particular, we consider whether growth can alter the material behaviour as to allow for collapse.

A fundamental aspect of the theory of elastic growth is the creation of residual stress through differential growth (inhomogeneous or anisotropic). As a body grows differentially, it is subjected to an elastic response ensuring compatibility and integrity of the material. This elastic response generates the so-called "residual stress". It has been shown that growth induced residual stress can have both stabilizing and destabilizing effects [2]. These stresses are common in many biological structures, and play an important role in the mechanics of growing elastic bodies $[19,20]$. Similarly, we show here that for the anticavitation problem, the development of residual stress serves as a primary force in opposition to the full collapse of the void.

For simplicity and to allow for analytical progress, we consider here a spherically symmetric shell, and ask whether growth may be imposed upon the shell such that it deforms to a solid sphere. These ideas can be easily generalised to cylindrical shells. We begin with the formulation of the problem through nonlinear elasticity, adding the component of growth via the formulation of multiplicative decomposition [16], in which the deformation tensor is given by the product of a growth tensor and an elastic strain tensor. The specific form of growth is captured by two functions (of radii) describing the amount of material that is added or removed in the radial and circumferential directions. We explore whether there exist functions that admit anticavitation. This is largely dependent on the strain energy function for the material. For a large class of materials, we show that anticavitation is in fact not possible for most physically relevant growth functions, but can be achieved in the proper limits when singular and/or vanishing growth functions are allowed. This analysis is shown to be relevant in the context of cumulative growth.

\section{Setup, regular growth}

We consider the symmetric growth and deformation of a spherical shell composed of an incompressible, hyperelastic material. The general question we explore is whether growth may be imposed such that the shell deforms to a solid sphere. Suppose that the inner and outer radii in the reference configuration are given by $R=A$ and $R=B$, respectively, and that the deformed shell is described in the current configuration by the function $r(R)$, which gives the radius of a sphere with initial radius $R$ in the reference configuration. Let $r(A)=a$ and $r(B)=b$, so that after deformation the shell in the current configuration has boundary radii 
$a$ and $b$. For this map, the geometric deformation tensor is given by $\mathbf{F}=\operatorname{diag}\left(r^{l}, r / R, r / R\right)$ [12], where primes denote differentiation with respect to $R$. The elastic strain tensor is $\mathbf{A}=$ $\operatorname{diag}\left(\alpha_{1}, \alpha_{2}, \alpha_{2}\right)$, and the growth is described by the growth tensor $\mathbf{G}=\operatorname{diag}\left(\gamma_{1}, \gamma_{2}, \gamma_{2}\right)$. For axisymmetric deformations, the $\alpha_{i}$ and $\gamma_{i}$ can be chosen to be functions only of $R$. Given the functions $\gamma_{1}, \gamma_{2}$, and external loading, the mechanical equilibrium determines the value of the function $r(R)$ and, in particular, the inner radius $a$.

The question of void collapse for a growing elastic material is then: What are the conditions on the growth functions $\gamma_{i}(R)$ and external loading so that there exists a solution of mechanical equilibrium with $a=0$ ?

Using the multiplicative decomposition $\mathbf{F}=\mathbf{A} \cdot \mathbf{G}$ in combination with the elastic imcompressibility condition $\operatorname{det}(\mathbf{A})=1$, we obtain the relations $\alpha_{1}=\alpha^{-2}$ (where we have denoted $\left.\alpha:=\alpha_{2}\right), r^{\prime}=\gamma_{1} / \alpha^{2}$, and $r / R=\alpha \gamma_{2}$. From these, the deformation may be written as

$$
r^{3}-a^{3}=3 \int_{A}^{R} \gamma_{1}(R) \gamma_{2}^{2}(R) R^{2} d R .
$$

Hyperelasticity implies that there exists a strain energy function $W=W(\mathbf{A})$. Letting $\mathbf{T}$ denote the Cauchy stress tensor, the stress-strain relation is $\mathbf{T}=\mathbf{A} \cdot W_{\mathbf{A}}-p \mathbf{1}$, where $p$ is a Lagrange multiplier representing hydrostatic pressure. Denote the non-vanishing components of the Cauchy stress tensor by $t_{1}=T_{11}$, the radial stress, and $t_{2}=T_{22}=T_{33}$, the hoop stress. In terms of these variables, the stress-strain relationship is $t_{1}=\alpha_{1} W_{1}-p, t_{2}=\alpha_{2} W_{2}-p$, where $W_{i}=\frac{\partial W}{\partial \alpha_{i}}$. Mechanical equilibrium requires $\operatorname{div}(\mathbf{T})=0$, where div is the divergence in the current configuration - the only non-vanishing equation is

$$
\frac{\partial t_{1}}{\partial r}+\frac{2}{r}\left(t_{1}-t_{2}\right)=0
$$

and from this we obtain a closed equation for the radial stress (see [2] for details):

$$
\frac{\partial t_{1}}{\partial r}=\frac{\alpha}{r} \hat{W}^{\prime}(\alpha)
$$

Here we have introduced the auxiliary function $\hat{W}(\alpha)=W\left(\alpha^{-2}, \alpha, \alpha\right)$. Integrating Equation (3), and defining $P=t_{1}(A)-t_{1}(B)$ as the applied load on the shell, we have

$$
-P=\int_{a}^{b} \frac{\alpha \hat{W}^{\prime}}{r} d r
$$

A typical procedure is to prescribe the growth functions $\gamma_{i}(R)$ and an applied load. Then, Equation (4) may be thought of as defining a relation to solve for the inner radius $a$, since the outer radius $b$ is a function of $a$ via Equation (1). Note that the functions $\gamma_{i}$ are implicitly embedded in (4) through Equation (1) as well as through the relation $r / R=\alpha \gamma_{2}$. Once $a$ is known, the deformation is completely determined.

Void collapse depends on the convergence of the integral found in Equation (4) in the limit $a \rightarrow 0$. If the integral converges, then the value of the integral gives the applied load necessary for anticavitation. The response of the shell to a given growth law, and thus whether or not anticavitation can be achieved, depends on the material properties, encompassed in the form of the strain energy function $\hat{W}$. There are many different strain energy functions available in the literature. In cavitation problems, Ball showed that it is the behavior of $\hat{W}(\alpha)$ as $\alpha \rightarrow \infty$ that determines whether the material admits cavitation. In contrast, Abeyaratne and Hou showed that the behavior of $\hat{W}$ as $\alpha \rightarrow 0$ determines void collapse. 
Here we show that with the addition of growth, the behavior of the auxiliary function both at zero and infinity plays a role in anticavitation. We classify the possibility of anticavitation for strain energy functions $\hat{W}$ with power law behavior as $\alpha$ approaches zero and infinity. Here, we use the notation

$$
f(x) \sim g(x) \text { as } x \rightarrow x_{0} \text { if } \lim _{x \rightarrow x_{0}} \frac{f(x)}{g(x)}=c \neq 0, c \text { a constant }
$$

and restrict our attention to strain energy functions which satisfy the following hypothesis:

Hypothesis 1 The auxiliary function $\hat{W}(\alpha)$ associated with the strain energy function $W$ for an incompressible, hyperelastic material satisfies

$$
\hat{W}(\alpha) \sim \alpha^{m_{0}} \text { as } \alpha \rightarrow 0, \hat{W}(\alpha) \sim \alpha^{m_{\infty}} \text { as } \alpha \rightarrow \infty
$$

for some constants $m_{0}$ and $m_{\infty}$.

Along with the behavior of the strain energy, we will need to characterize the behavior of the growth functions $\gamma_{i}$. Define a regular growth function as one that is finite, continuous, and strictly positive. We will say that a material undergoes a regular growth when the growth functions satisfy these requirements. As a first case, we have the following:

Proposition 1 A material undergoing regular growth admits spherically symmetric anticavitation if and only if $\hat{W}(\alpha)$ is bounded as $\alpha \rightarrow 0$.

Proof: Recall that $\alpha=\frac{r}{\gamma_{2} R}$. Hence, as $r$ goes to zero, so does $\alpha$. By assumption, $\hat{W} \sim \alpha^{m_{0}}$ as $\alpha \rightarrow 0$. Then

$$
\frac{\alpha \hat{W}^{\prime}}{r} \sim \frac{\alpha \alpha^{m_{0}-1}}{r} \sim \frac{1}{r^{1-m_{0}}} \text { as } r \rightarrow 0 .
$$

Hence,

$$
\int_{0}^{b} \frac{\alpha \hat{W}^{\prime}}{r} d r
$$

will converge if and only if $m_{0}>0$, i.e. if and only if $\hat{W}$ is bounded as $\alpha \rightarrow 0$.

The condition that $\hat{W}$ must be bounded as $\alpha \rightarrow 0$ coincides with the condition for void collapse given by Abeyaratne and Hou in the absence of growth. Hence, imposing a regular growth function cannot change the property of a material to admit anticavitation. However, for materials that satisfy this condition, void collapse can occur in the absence of external loading but through growth-induced residual stress. To illustrate this phenomenon, consider a material with strain energy $W=\frac{\mu}{\beta}\left[\left(1+\beta\left(\alpha_{1}^{2}+\alpha_{2}^{2}+\alpha_{3}^{2}-3\right)\right)^{n}-1\right]$, where $\mu>0, \beta>0$, and $n$ are material constants as suggested in [1]. This strain energy function admits anticavitation for $n<0$. We consider homogeneous, anisotropic growth for an unloaded shell by setting $\gamma_{2}=1, \gamma_{1}>1$ a constant, and the external pressure $P=0$. Figure 1 plots the inner radius $a$ as a function of $\gamma_{1}$ for initial radii $A=1, B=2$, and parameters $\mu=\beta=1$. There is a critical value of $\gamma_{1}$ for which the cavity collapses. Above this value, the inner radius is still zero, with a finite compressive stress induced at the origin due to the contact. 


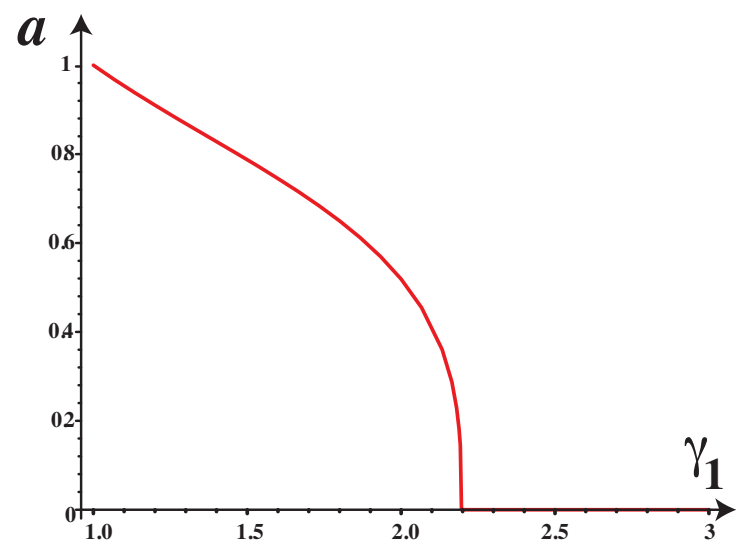

Fig. 1 Anticavitation of a shell (with initial radii $A=1$ and $B=2$ ) induced by radial growth. The inner radius in the current configuration, $a$, is plotted as a function of the radial growth $\chi$ for $\gamma_{2}=1, \mu=\beta=1, n=-1$.

\section{Diverging/vanishing $\gamma_{i}$}

The condition that $\hat{W}$ be bounded as $\alpha$ goes to zero is very restrictive. For most materials, with $\hat{W}$ unbounded as $\alpha \rightarrow 0$, anticavitation requires infinite external pressure. However, we have assumed that the functions $\gamma_{i}$ are finite and strictly positive. As is discussed in Appendix A, divergent growth functions can arise naturally in a cumulative growth process where growth is localised close to the boundary. Therefore, we consider materials for which the strain energy is unbounded as $\alpha$ approaches zero, and explore whether anticavitation is possible with diverging and/or vanishing $\gamma_{i}$

Following the assumptions and notations of the previous section, we write Equation (3) in terms of $R$, which gives

$$
\frac{\partial t_{1}}{\partial R}=\frac{\hat{W}^{\prime}(\alpha) \gamma_{1}}{\alpha^{2} \gamma_{2} R} .
$$

Note that since we have assumed that $\hat{W}$ is unbounded as $\alpha \rightarrow 0$ and the strain energy $W$ is non-negative for all deformations, we have $\lim _{\alpha \rightarrow 0} \hat{W}=+\infty$. Now, we integrate from $R=A$ to $B$ and define the function

$$
G(a):=\int_{A}^{B} \frac{\partial t_{1}}{\partial R}(R ; a) d R .
$$

For a given external pressure $P$, the positive root of $G(a)+P=0$ gives the inner radius. To focus on the effect of growth alone, we consider shells with no external pressure. Hence, we set $P=0$ and explore the convergence or divergence of the integral $G(0)$. We allow the $\gamma_{i}$ to either vanish or blow up at the inner boundary. We define $y:=R-A$ and assume that as $y \rightarrow 0$ (that is $R \rightarrow A$ ) we have

$$
\gamma_{1} \sim y^{-p_{1}}, \quad \gamma_{2} \sim y^{-p_{2}}
$$

where $p_{1}$ and $p_{2}$ are real numbers. From (1) with $a=0$, we find that

$$
r^{3} \sim y^{1-p_{1}-2 p_{2}} \text { as } R \rightarrow A
$$


For the map $r(R)$ to be well defined, we therefore require that $1-p_{1}-2 p_{2}>0$. Next, using the relation $\alpha=r /\left(\gamma_{2} R\right)$, we have $\alpha \sim y^{\left(1-p_{1}+p_{2}\right) / 3}$. The behavior of $G(0)$ will depend on whether $\alpha$ is vanishing or diverging as $R \rightarrow A$. Thus, from here we divide the problem into 3 cases, based on the sign of the quantity

$$
J:=1-p_{1}+p_{2}
$$

3.0.1 Case 1: $J>0$

If $J>0$, then $\alpha \rightarrow 0$ as $R \rightarrow A$. Since by assumption $\hat{W} \sim \alpha^{m_{0}}$ as $\alpha \rightarrow 0, m_{0}<0$. In this case, we have

$$
\frac{\partial t_{1}}{\partial R} \sim y^{E_{1}} \text { as } y \rightarrow 0
$$

where $E_{1}=\frac{J}{3}(m+0-3)-p_{1}+p_{2}$. The requirement for the boundedness of the radial stress is $E_{1}>-1$. This reduces to $J m_{0}>0$ which cannot hold since $J>0$ and $m_{0}<0$.

3.0.2 Case 2: $J<0$

For $J<0, \alpha \rightarrow \infty$ as $R \rightarrow A$, and we consider the behavior of $\hat{W}$ as $\alpha \rightarrow \infty$. For isotropic material, $W$ has the symmetry $W\left(\alpha_{1}, \alpha_{2}, \alpha_{2}\right)=W\left(\alpha_{2}, \alpha_{1}, \alpha_{1}\right)$. Thus for $\hat{W}(\alpha)=W\left(\alpha^{-2}, \alpha, \alpha\right)$, it follows that $\lim _{\alpha \rightarrow 0} \hat{W}(\alpha)=\lim _{\alpha \rightarrow \infty} \hat{W}(\alpha)=\infty$, and we conclude that $\hat{W}$ is unbounded at infinity. Therefore, we write $\hat{W} \sim \alpha^{m_{\infty}}$ as $\alpha \rightarrow \infty$, with $m_{\infty}>0$. As in Case 1,

$$
\frac{\partial t_{1}}{\partial R} \sim y^{E_{1}} \text { as } y \rightarrow 0
$$

and again, convergence requires $E_{1}>-1$, which simplifies to $J m_{\infty}>0$ which again does not hold based on the assumptions of $J$ and $m_{\infty}$.

3.0.3 Case 3: $J=0$

If $J=0, \alpha$ and $\hat{W}^{\prime}(\alpha)$ behave like constants as $R \rightarrow A$ and hence play no role in the convergence or divergence of the integral. Here,

$$
\frac{\partial t_{1}}{\partial R} \sim y^{-p_{1}+p_{2}} \text { as } y \rightarrow 0
$$

but the requirement for convergence is equivalent to requiring $J<0$, which does not hold.

From these 3 cases, we conclude that even if we allow the growth functions to diverge or vanish at the inner boundary, we still cannot send the inner radius $a$ to zero. To summarize:

Proposition 2 Let $\gamma_{i} \sim(R-A)^{-p_{i}}$ as $R \rightarrow A, i=1,2$, be radial growth functions. If $\hat{W}$ is unbounded as $\alpha \rightarrow 0$, the material does not admit anticavitation for any real numbers $p_{1}$ and $p_{2}$. 


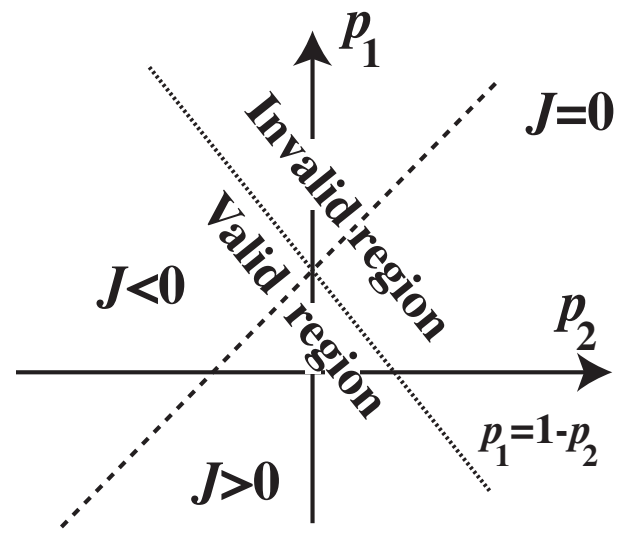

Fig. 2 Depiction of $p_{1}-p_{2}$ plane. The line $p_{1}=1-2 p_{2}$ divides the plane into a valid and invalid region. The plane is further divided based on the sign of the quantity $J$.

3.1 The $p_{1}-p_{2}$ plane - filling the void

The previous proposition seems to point to the impossibility of anticavitation in locally unbounded strain energy-functions. However, in this section we show that the void can be made arbitrarily small with the proper balance of diverging/vanishing growth. To do this, we consider more closely the relationship between the rates of growth, captured by the exponents $p_{1}$ and $p_{2}$ in the relation (11). Figure 2 depicts the $p_{1}-p_{2}$ plane. The requirement for a well defined map $r(R)$ is that $p_{1}<1-2 p_{2}$, which divides the plane into a valid and invalid region. We further divide the plane based on the sign of the quantity $J$. For any choice of $p_{1}$ and $p_{2}, G(0)$ is a diverging integral. The following result establishes the direction of divergence of this integral (plus or minus infinity).

Proposition 3 Assume that $\hat{W}(\alpha)$ is unbounded as $\alpha \rightarrow 0$. Then, for $J \neq 0$,

$$
J \lim _{a \rightarrow 0^{+}} G(a)=-\infty .
$$

That is, the direction of divergence (plus or minus infinity) of the integral $G(a)$ given in Equation (10) in the limit $a=0$ is determined by the sign of $J$.

Proof: We use again the symmetry of $W$ for isotropic materials: $W\left(\alpha_{1}, \alpha_{2}, \alpha_{2}\right)=W\left(\alpha_{2}, \alpha_{1}, \alpha_{1}\right)$,. Thus for $\hat{W}(\alpha)=W\left(\alpha^{-2}, \alpha, \alpha\right)$, we have $\lim _{\alpha \rightarrow 0} \hat{W}(\alpha)=\lim _{\alpha \rightarrow \infty} \hat{W}(\alpha)=\infty$. Since $\alpha \rightarrow 0$ as $R \rightarrow A$ when $J>0$ and $\alpha \rightarrow \infty$ as $R \rightarrow A$ when $J<0$ and since all other terms in $G(0)$ are non-negative, the result follows.

Given this change in divergence based on the sign of $J$, the following theorem establishes that a solution may be found with an arbitrarily small void.

Theorem 1 Let $\hat{W}(\alpha)$ be unbounded as $\alpha \rightarrow 0$, and assume that the growth functions are such that $\gamma_{i} \sim(R-A)^{-p_{i}}$ as $R \rightarrow A$. Then for any fixed $p_{2}<0$ and $\varepsilon>0$, there exists $p_{1}$ for which the unloaded spherical shell has an inner radius $a<\varepsilon$.

Proof: Let $\varepsilon>0$ and $p_{2}<0$ be fixed. Since the function $G(a)$ depends on the exponent $p_{1}$, we define for each $p_{1}$ the function $H\left(a, p_{1}\right)=G(a)$. Further, there is a distinguished 


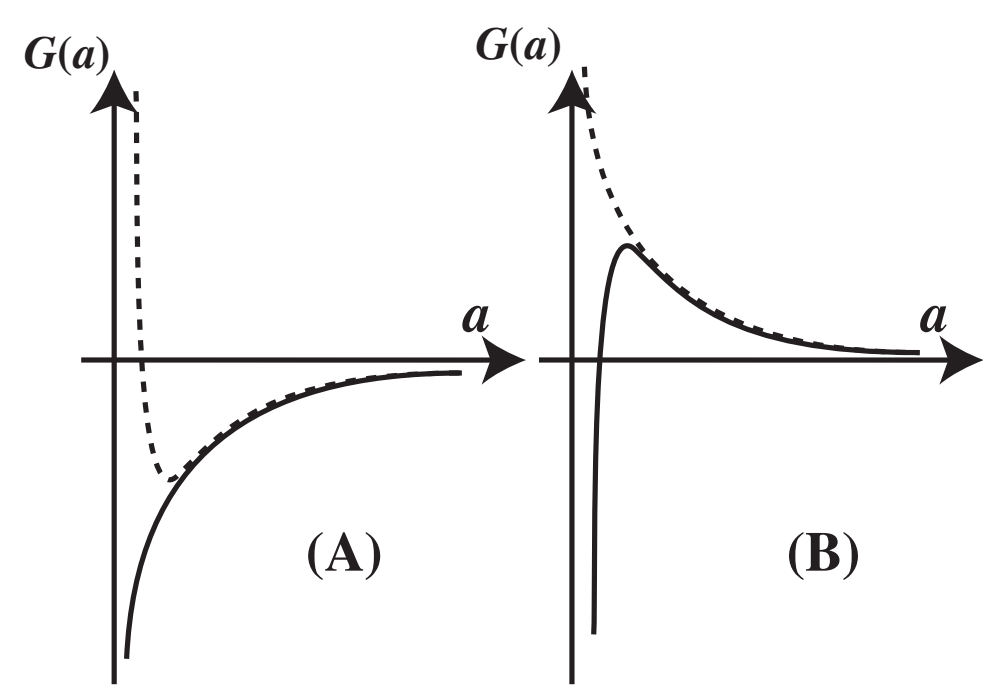

Fig. 3 Depiction of the local behavior of $G(a)$ near $a=0$. The dashed (solid) lines represent $G(a)$ directly above (below) $J=0$. The change in sign of divergence ensures a root arbitrarily close to zero.

exponent $p_{1}^{*}=1+p_{2}$ for which $J=0$. Then, following Case 3 above, we have,

$$
\lim _{a \rightarrow 0^{+}} H\left(a, p_{1}^{*}\right)=s\left(p_{2}\right) \infty
$$

where $s\left(p_{2}\right)$ is either +1 or -1 depending on the value of $p_{2}$ and the strain-energy function. We consider here the case where $s\left(p_{2}\right)=+1$, as the case where $s\left(p_{2}\right)=-1$ follows with appropriate sign changes. Since $H$ is continuous with respect to a, we can choose $0<a_{1}<\varepsilon$ such that $\phi:=H\left(a_{1}, p_{1}^{*}\right)>0$. For fixed $a_{1}>0, H$ is a continuous function of $p_{1}$. Hence there exists $\delta>0$ such that

$$
\left|p_{1}^{*}-p_{1}\right|<\delta \Rightarrow\left|H\left(a_{1}, p_{1}^{*}\right)-H\left(a_{1}, p_{1}\right)\right|<\phi .
$$

Let $\hat{p}_{1}=p_{1}^{*}-\delta / 2$; then $H\left(a_{1}, \hat{p}_{1}\right)>0$. Further, since $\hat{p}_{1}<p_{1}^{*}$, we have

$$
\lim _{a \rightarrow 0^{+}} H\left(a, \hat{p}_{1}\right)=-\infty .
$$

Again by continuity of $H$ as a function of a, there exists $a^{*}>0$ such that $H\left(a, \hat{p}_{1}\right)<0 \forall a<$ $a^{*}$. Therefore, we can choose $0<a_{2}<a_{1}$ such that $H\left(a_{2}, \hat{p}_{1}\right)<0$ and by the Intermediate Value Theorem there exists $a_{3} \in\left(a_{2}, a_{1}\right)$ such that $H\left(a_{3}, \hat{p}_{1}\right)=0$. Since by construction $a_{3}<\varepsilon$, the result follows.

The above argument is illustrated in Figure 3, where the local shape of the curve $G(a)$ near $a=0$ will transition according to one of two possibilities. In Figure 3, the dashed lines represent the shape of the curve directly above the line $J=0$ and diverge to infinity, while the solid lines represent the curve directly below $J=0$, with the divergence to minus infinity. In case (A), the void may be made arbitrarily small by approaching the line $J=0$ from above. In (B), the same is done by approaching from below.

Which scenario is appropriate, i.e. whether to approach from above or below, depends on the behavior of $G(0)$ on the line $J=0$. When $J=0, \alpha$ approaches a constant as $R \rightarrow A$, 
and the direction of divergence will depend on the particular strain energy function and can also change along the line $J=0$. If, at a particular point on the line $J=0, G(0)$ diverges to positive infinity, then that point corresponds to case (B), and the root may be sent to zero by approaching $J=0$ from below. If instead $G(0)$ diverges to negative infinity, the point corresponds to case (A) and $J=0$ should be approached from above.

The analysis may be interpreted as follows: the region of the plane where $J>0$ corresponds to an infinite circumferential growth, while $J<0$ corresponds to an infinite radial growth. Anticavitation is achieved by approaching the boundary between these two regions, i.e. by finding a balance between radial and circumferential growth.

\section{Stability}

We have shown that a root of $G(a)$ may be brought arbitrarily close to zero. This means that there exists a mechanical solution of the problem with an arbitrarily small void. However, we have not demonstrated that these solutions can be reached and are stable. In the transition crossing the line $J=0$, multiple roots may occur, only one of which may be stable. A stability analysis for symmetric deformations can be performed by considering the minima of the potential energy.

Theorem 2 Let $a>0$ be a solution of $G(a)=0$ corresponding to the inner radius of an equilibrium solution in the set of radially symmetric deformations. Then, the associated deformation is locally stable if $G^{\prime}(a)>0$, and locally unstable if $G^{\prime}(a)<0$.

Proof: The potential energy for the deformation, as a function of the inner radius a, is found by integrating the strain energy function over the shell; that is

$$
U(a)=\int_{a}^{b} \hat{W}(\alpha) 4 \pi r^{2} d r=\int_{A}^{B} \hat{W}(\alpha) \gamma_{1} \gamma_{2}^{2} 4 \pi R^{2} d R
$$

Taking a derivative with respect to $a$, and using the relationships $\alpha=r /\left(\gamma_{2} R\right)$ and $r^{3}=$ $a^{3}+\int_{A}^{R} \gamma_{1} \gamma_{2}^{2} R^{2} d R$ as well as the connections (9), (10), we obtain

$$
U^{\prime}(a)=4 \pi a^{2} G(a)
$$

Taking a second derivative, and using the fact that $G(a)=0$ at a point of equilibrium, we have $U^{\prime \prime}(a)=4 \pi a^{2} G^{\prime}(a)$, and the result follows by the concavity of the energy potential.

Whether or not anticavitation is possible depends on whether or not there are multiple roots, which depends on the form of the curve $G(a)$, which in turn depends on the choice of strain energy function. As an example, consider a Neo-Hookean material, characterized by strain energy function $W=\mu\left(\alpha_{1}^{2}+\alpha_{2}^{2}+\alpha_{3}^{2}-3\right)$, where $\mu>0$, so that $\hat{W}=\mu\left(\alpha^{-4}+\right.$ $\left.2 \alpha^{2}-3\right)$.

In Figure 4, the curve $G(a)$ is plotted for a sequence of points in the $p_{1}-p_{2}$ plane, where $\gamma_{1}=(R-A)^{-p_{1}}, \gamma_{2}=(R-A)^{-p_{2}}$, and parameters $A=1, B=2$, and $\mu=1$. The 5 curves in (B) correspond to the 5 points in (A). As the line $J=0$ is approached from below, the single root of the curve approaches zero. Since there is only a single root, anticavitation is possible in the limit of $p_{1}$ approaching 0.5 from the left (in this example $p_{2}=-0.5$ is fixed). Above the line (point 5), the divergence at zero flips, and the root disappears.

In Figure 5, $p_{2}=-1$ is fixed. In (A), $G(a)$ is plotted on both sides of $J=0$. For $p_{1}=$ -0.06 , there is a root, so that crossing the line $J=0$ to the point $p_{1}=0.06$ yields a second 


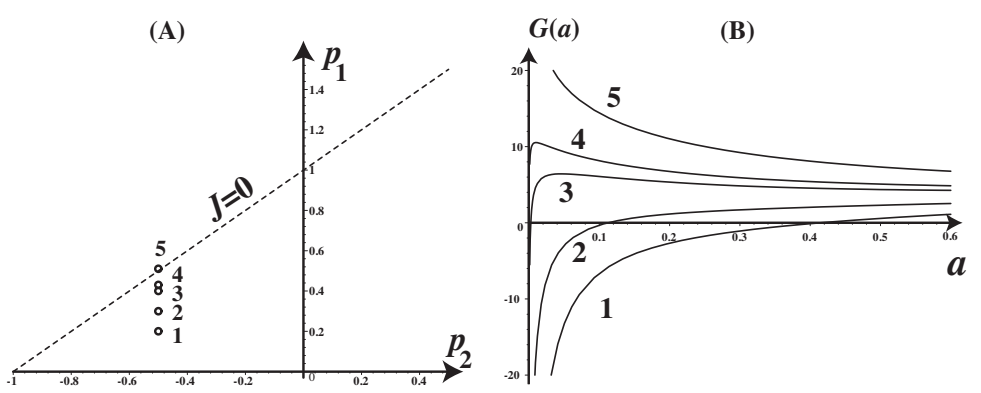

Fig. 4 (A) 5 points in the $p_{1}-p_{2}$ plane defining 5 choices of $\gamma_{i}$. (B) The corresponding curves $G(a)$ for a Neo-Hookean material. Anticavitation is achieved as $J=0$ is approached from below.

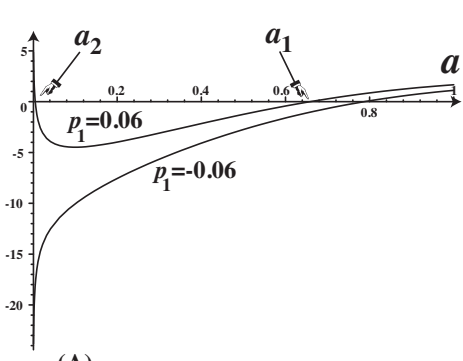

(A)

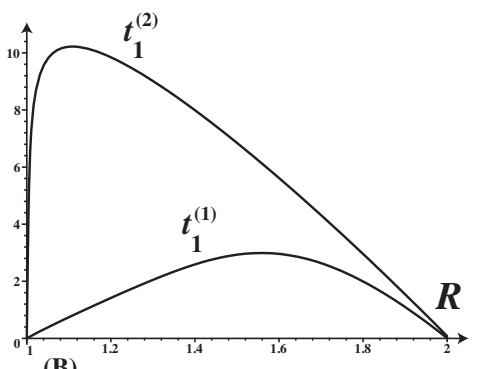

(B)

Fig. 5 (A) The curve $G(a)$ above $\left(p_{1}=0.06\right)$ and below $\left(p_{1}=-0.06\right) J=0$ for $p_{2}=-1$. (B) The radial stress for the two roots $a_{1}$ and $a_{2}$ from (A).

root, an unstable solution. In (B), the residual radial stress is plotted for each of these two roots, $a_{1}$ and $a_{2}$. For both solutions, the residual stress is tensile, and as expected, the stress is much higher in the unstable solution with root $a_{2}$.

It is worth noting that the Neo-Hookean material used in this example does not satisfy the criterion of Abeyaratne and Hou for anticavitation given in [1], i.e. $\hat{W}$ is unbounded as $\alpha \rightarrow 0$. In other words, external pressure on the shell cannot cause void collapse, whereas growth can cause the void to become arbitrarily small.

\section{Conclusion}

In this paper, we explored anticavitation in a growing elastic shell as a function of the form and rate of imposed growth. The question of whether void collapse is possible is formulated in terms of the convergence or divergence of a single integral, given in Equation (4). Our analysis consists in determining radial growth functions for which convergence of this integral is achieved. We concluded that the integral will always diverge, even for growth functions which diverge or vanish at the point where the integral is improper. Nevertheless, based on the rates at which the growth functions diverge/vanish, the void may still be made arbitrarily small. This can only be achieved in the proper limits of the rates at which the growth functions diverge/vanish, illustrating the fine balance of radial and circumferential growth needed for anticavitation. Note, however, that we have only considered strain energy 
functions with power law behavior. An extension of the present work would be to study functions $\hat{W}(\alpha)$ which are either bounded by power laws or diverge faster than any power, in which case it is likely that exponentially diverging/vanishing growth functions would also be necessary.

The effect of growth on material properties is clear - anticavitation is possible through growth for materials for which it is impossible through applied pressure alone. Furthermore, the analysis presented in the Appendix suggests that diverging or vanishing growth functions naturally arise in the context of cumulative growth.

The present study of void collapse reveals that filling a void through a purely elastic mechanism in physical or biological systems requires a delicate balance between growth laws and material parameters as to achieve arbitrarily small voids. In practice, it would involve either a control mechanism or other mechanisms such as accretion at the inner boundary or asymmetric deformation of the body. Nonetheless, from a mechanical standpoint, the simplified model presented here illustrates the important role of differential growth in anticavitation processes.

Acknowledgments: This publication is based on work supported by Award No. KUK-C1013-04 , made by King Abdullah University of Science and Technology (KAUST), and based in part upon work supported by the National Science Foundation under grants DMS0907773 (AG)

\section{Appendix: Cumulative growth}

We have analyzed the growth of a spherical shell with radial growth functions which either diverged or vanished at the inner edge $R=A$. As a single growth step, this may appear unphysical. However, this single step should be seen as the result of a cumulative growth process. In a continuously growing body, the growth can be modeled by a relation of the form

$$
\dot{\mathbf{G}}=H(\mathbf{G}, \mathbf{A}, \mathbf{T}, \ldots, ; \mathbf{X}, t) ;
$$

where the rate of growth is not necessarily constant, and might in general depend on the growth and strain tensors, tension, position, time, and potentially other factors. This can be approximated by a discrete growth process, where (23) is replaced by $\mathbf{G}(t+\Delta t)=\mathbf{G}(t)+$ $\Delta t H$. This relation may be seen to define an incremental growth, $\mathbf{G}_{\mathrm{inc}}:=\mathbf{G}(t+\Delta t)-\mathbf{G}(t)$. If, at each incremental step, the elastic response is captured by $\mathbf{A}_{\text {inc }}$, then through multiplicative decomposition we can define an incremental deformation $\mathbf{F}_{\text {inc }}=\mathbf{A}_{\text {inc }} \cdot \mathbf{G}_{\text {inc }}$. Thus, at each step, the material grows according to some incremental growth law, followed by an elastic response necessary for compatibility. For each incremental step, one can define a total growth tensor, such that the $i^{\text {th }}$ incremental step is equivalent to a single step with a deformation tensor defined from the initial, stress free configuration (see [7] for details).

In the spherical geometry we have considered here, let $R$ be the radius in the initial configuration and $r_{i-1}$ the radius in the current configuration after the $(i-1)^{\text {th }}$ step. Then the incremental growth tensor for the $i^{\text {th }}$ step will be of the form

$$
\mathbf{G}_{\text {inc }}^{(i)}=\operatorname{diag}\left(\gamma_{1}^{(i)}\left(r_{i-1}\right), \gamma_{2}^{(i)}\left(r_{i-1}\right), \gamma_{2}^{(i)}\left(r_{i-1}\right)\right) .
$$

The total deformation defines a map $r_{i-1}=r_{i-1}(R)$, and so the $\gamma$ are functions of the radius in the initial configuration, $R$. The total growth tensor is [7] 

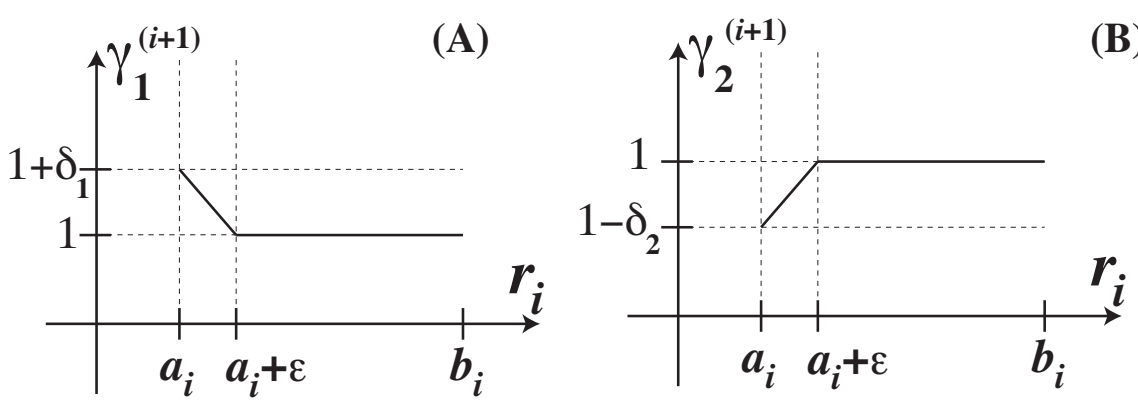

(B)

Fig. 6 Piecewise incremental growth laws in a cumulative growth process.

$$
\mathbf{G}^{(i)}=\operatorname{diag}\left(\Pi_{n=1}^{i} \gamma_{1}^{(n)}(R), \Pi_{n=1}^{i} \gamma_{2}^{(n)}(R), \Pi_{n=1}^{i} \gamma_{2}^{(n)}(R)\right)
$$

If the shell is growing in a continuous process such that growth is always focused on the inner edge, then the incremental $\gamma$ will take extreme values at the inner edge, corresponding to $R=A$. Therefore, the total growth functions, which are products of the growth functions of all previous steps, will be well approximated by the diverging/vanishing $\gamma_{i}$ we have considered in this paper.

In this process, the possibility of anticavitation will depend on the specific form of the incremental growth laws, encompassed by a relation of the form (23). As a simple example, consider a Neo-Hookean material subject to the piecewise linear growth functions pictured in Figure 6.

At each incremental step, the region over which growth/resorption is restricted is given by the parameter $\varepsilon$, while the $\delta_{i}$ dictate the rate or amount of growth/resorption. Figure 7 shows the results of the cumulative growth process for the parameters $\delta_{1}=0.6, \delta_{2}=0.3$, and $\varepsilon=0.5$. Here, the balance of radial growth and circumferential resorption is such that the growth collapses the void, as seen in Figure 7(A). The total growth function $\gamma_{1}(R)$ at the $9^{\text {th }}$ step is plotted in Figure 7(B), illustrating the blow-up like behavior of the radial growth function after several steps.

As would be expected from the analysis of Section 3, a small change in the growth rate parameters $\delta_{i}$ can cause anticavitation to be no longer possible (plots not included).

\section{References}

1. R. Abeyaratne and H-S Hou. Void collapse in an elastic solid. J. Elasticity, 26:23-42, 1991.

2. M. Ben Amar and A. Goriely. Growth and instability in elastic tissues. J. Mech. Phys. Solids, 53:2284 2319, 2005.

3. J. M. Ball. Discontinuous equilibrium solutions and cavitation in nonlinear elasticity. Phil. Trans. Roy. Soc. London A, 306:557-610, 1982.

4. G. K. Batchelor. An Introduction to Fluid Mechanics. Cambridge University Press, Cambridge, 1967.

5. J. Dollhofer, A. Chiche, V. Muralidharan, C. Creton, and C. Y. Hui. Surface energy effects for cavity growth and nucleation in an incompressible neo-hookean material - modeling and experiment. Int. J. Solids Structures, 41:6111-6127, 2004.

6. A. N. Gent and P. B. Lindley. Internal rupture of bonded rubber cylinders in tension. Proc. Roy. Soc. London A, 249:195-205, 1958. 

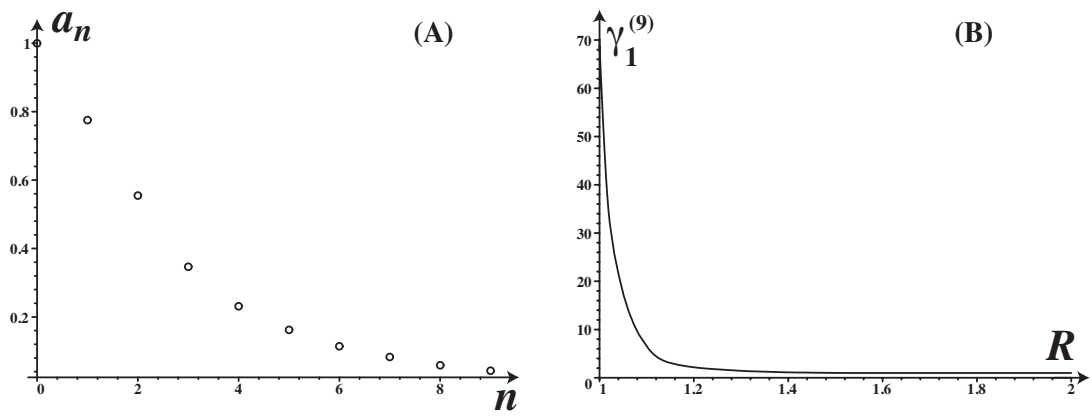

Fig. 7 Cumulative growth process in a Neo-Hookean shell. (A) - Progression of the inner radius through 9 steps of growth. (B) The total radial growth function for the gh $^{\text {h }}$ step of growth. The incremental growth functions are as pictured in Figure 6 with $\delta_{1}=0.6, \delta_{2}=0.3$, and $\varepsilon=0.5$.

7. A. Goriely and M. Ben Amar. On the definition and modeling of incremental, cumulative, and continuous growth laws in morphoelasticity. Biomech. Model. Mechanobiol., 6(5):289-296, 2007.

8. A. Goriely, D. E. Moulton, and R. Vandiver. Elastic cavitation and differential growth in biological tissues. Preprint, 2010.

9. C. O. Horgan and D. A. Polignone. Cavitation in nonlinearly elastic solids: A review. Appl. Mech. Rev., 48(8):471-485, 1995.

10. O. Lopez-Pamies. Onset of cavitation in compressible, isotropic, hyperelastic solids. J. Elasticity, 94:115-145, 2009.

11. J. McMahon, A. Goriely, and M. Tabor. Spontaneous cavitation in growing elastic membranes. Math. Mech. Solids, doi:10.1177/1081286508092010(8):471-485, 2008.

12. R. W. Ogden. Non-linear Elastic Deformation. Dover, New York, 1984.

13. T. J. Pence and H. Tsai. Swelling induced cavitation of elastic spheres. Math. Mech. Solids, 11:527-551, 2006.

14. K. A. Pericak-Spector, J. Sivaloganathan, and S. J. Spector. An explicit radial cavitation solution in nonlinear elasticity. Math. Mech. Solids, 7(1):87-93, 2002.

15. D. A. Polignone and C. O. Horgan. Cavitation for incompressible anisotropic nonlinearly elastic spheres. J. Elasticity, 33:27-65, 1993.

16. E. K. Rodriguez, A. Hoger, and A. McCulloch. Stress-dependent finite growth in soft elastic tissues. $J$. Biomech., 27:455-467, 1994.

17. J. Sivaloganathan. On cavitation and degenerate cavitation under internal hydrostatic pressure. Proc. Roy. Soc. London A, 455:3645-3664, 1999.

18. J. Sivaloganathan and S. J. Spector. On cavitation, configurational forces and implications for fracture in a nonlinearly elastic material. J. Elasticity, 67:25-49, 2002.

19. R. Skalak, S. Zargaryan, R. K. Jain, P. A. Netti, and A. Hoger. Compatibility and the genesis of residual stress by volumetric growth. J. Math. Biology, 34:889-914, 1996.

20. R. Vandiver and A. Goriely. Tissue tension and axial growth of cylindrical structures in plants and elastic tissues. Europhys. Lett. (EPL), 84(58004), 2008. 



\section{RECENT REPORTS}

33/09 Nematic liquid crystals : from Maier-Saupe to a continuum theory

Ball

Majumdar

34/09 Tangent unit-vector fields: nonabelian homotopy invariants and

Majumdar the Dirichlet energy

Robbins

Zyskin

35/09 A metabolite-sensitive, thermodynamically-constrained model of

Tran cardiac cross-bridge cycling: Implications for force development during ischemia

Smith

Loiselle

Crampin

36/09 Modelling bacterial behaviour close to a no-slip plane boundary: the influence of bacterial geometry

Shum

Gaffney

Smith

37/09 Optimal L2-error estimates for the semidiscrete Galerkin approx-

Goswami imation to a second order linear parabolic initial and boundary value problem with nonsmooth initial data

38/09 Optimal L2 estimates for semidiscrete Galerkin methods for parabolic integro-differential equations with nonsmooth data

Pani

Goswami

Pani

Yadav

39/09 Spatially structured oscillations in a two-dimensional excitatory neuronal network with synaptic depression

Kilpatrick

Bressloff

40/09 Stationary bumps in a piecewise smooth neural field model with synaptic depression

Kilpatrick

Bressloff

41/09 Homogenization for advection-diffusion in a perforated domain Haynes

Hoang

Norris

Zygalakis

42/09 Fast stochastic simulation of biochemical reaction systems by al- Melykuti ternative formulations of the Chemical Langevin Equation

Burrage

Zygalakis

43/09 Pseudoreplication invalidates the results of many neuroscientific Lazic studies

44/09 Cardiac cell modelling: Observations from the heart of the cardiac physiome project

45/09 A Hybrid Radial Basis Function - Pseudospectral Method for Thermal Convection in a 3-D Spherical Shell

Wright

Flyer

46/09 Refining self-propelled particle models for collective behaviour Yates

Baker

Erban

Maini 
47/09 Stochastic Partial Differential Equations as priors in ensemble methods for solving inverse problems

Potsepaev

Farmer

Aziz

48/09 DifFUZZY: A fuzzy spectral clustering algorithm for complex data sets

01/10 Fluctuations and instability in sedimentation

02/10 Determining the equation of state of highly plasticised metals from boundary velocimetry

03/10 Stability of bumps in piecewise smooth neural elds with nonlinear adaptation

04/10 Random intermittent search and the tug-of--war model of motordriven transport

05/10 Ergodic directional switching in mobile insect groups

06/10 Derivation of a dual porosity model for the uptake of nutrients by root hairs

07/10 Frost heave in compressible soils

08/10 A volume-preserving sharpening approach for the propagation of sharp phase boundaries in multiphase lattice Boltzmann simulations
Cominetti et al.

Guazzelli

Hinch

Hinch

Kilpatrick

Bressloff

Newby

Bressloff

Escudero et al.

Zygalakis

Roose

Majumdar

Peppin

Style

Sander

Reis

Dellar

Copies of these, and any other OCCAM reports can be obtained from:

Oxford Centre for Collaborative Applied Mathematics Mathematical Institute

24 - 29 St Giles'

Oxford

OX1 3LB

England

www.maths.ox.ac.uk/occam 